\title{
Cardiac Dysrhythmias and Anesthesia
}

\author{
Michelle Nacur Lorentz, TSA ${ }^{1}$, Bruna Silviano Brandão Vianna ${ }^{2}$
}

Summary: Lorentz MN, Vianna BSB - Cardiac Dysrhythmias and Anesthesia.

Background and objectives: Cardiac dysrhythmias are relatively common in the perioperative period and should be adequately diagnosed and treated by the anesthesiologist whenever indicated. The objective of this article was to review the most relevant aspects of cardiac dysrhythmias, as well as establishing the cause-effect relationship between drugs used in the perioperative period and dysrhythmias.

Contents: The mechanisms of dysrhythmias, drugs that can potentially cause dysrhythmias, besides diagnosis and treatment in the perioperative period are presented.

Conclusions: Perioperative dysrhythmias oftentimes do not require treatment and in others the treatment can generate iatrogenicity. Therefore, the knowledge of cardiac dysrhythmias and triggering factors allows a better approach of the perioperative period by the anesthesiologist avoiding wrong or unnecessary treatment.

Keywords: Anesthesia; Arrhythmias, Cardiac.

๑2011 Elsevier Editora Ltda. All rights reserved.

\section{INTRODUCTION}

\section{Cardiac dysrhythmias and anesthesia}

Dysrhythmias represent an important cause of perioperative complications because during this period there are several clinical situations that may trigger changes in cardiac rhythm ${ }^{1}$. These rhythm changes may be due to a primary etiology or to reversible causes that should be corrected. The prevalence of cardiac dysrhythmias varies according to the literature, type of surgical procedure, and the patient. In a multicenter study with 17,201 patients undergoing general anesthesia, dysrhythmias (tachycardia, bradycardia, or other dysrhythmias) were observed in $70.2 \%$, of which only $1.6 \%$ required treatment ${ }^{2}$. A large number of patients undergoing non-cardiac surgeries may develop dysrhythmias ${ }^{3,4}$ and the incidence of atrial fibrillation $(\mathrm{AF})$ is low after exploratory thoracotomy, but in elderly patients undergoing lobectomy, pneumectomy, and esophagogastrectomy the incidence rises to $12 \%$ to $33 \%{ }^{4}$.

Note that antidysrhythmic drugs can also cause dysrhythmias and oftentimes the anesthesiologist in an attempt

Received from Hospital Biocor, Belo Horizonte, MG, Brazil.

1. MSc from Universidade Federal de Minas Gerais (UFMG); Anesthesiologist of Hospital Biocor

2. R3 in Anesthesiology of Hospital Júlia Kubistchek

Submitted on September 8, 2010.

Approved on February 21, 2011.

Correspondence to:

Dra. Michelle Nacur Lorentz

Rua da Paisagem, 290, $6^{\circ}$ andar

Nova Lima

34000000 - Belo Horizonte, MG, Brazil

E-mail: mnacur@yahoo.com.br to treat perioperative dysrhythmia can cause iatrogenesis, and as such the knowledge of the physiology of the cardiac rhythm, anesthetic pharmacology, and risk-benefits of antidysrhythmic drugs is mandatory. Most perioperative dysrhythmias are benign, without significant hemodynamic consequences. Symptomatic patients, whose dysrhythmias can evolve to lifethreatening malignant dysrhythmias should be treated with antidysrhythmic drugs or electrotherapy ${ }^{5}$.

\section{Etiology}

In children the main causes of dysrhythmias include congenital cardiopathies, cardiomyopathies, and myocardial inflammatory disorders ${ }^{6}$. However, there are patients with premature ventricular contractions (PVCs) whose hearts are structurally normal. The incidence of benign PVCs has a biphasic distribution with a peak of approximately $15 \%$ in the first weeks of life decreasing to $<5 \%$ before adolescence, with a gradual increase in adolescents ${ }^{6}$. In the pediatric population with structurally normal myocardium sustained ventricular PVCs are relatively rare. Benign dysrhythmias usually have sinus rhythm, repolarization, normal ventricular function, and, in general, patients do not have a significant family history of mortality.

Infants and young children can present automatic atrial tachycardia (AAT), a rare supraventricular tachycardia. Postoperative (PO) tachycardia is relatively common in children and in most cases reflects sinus tachycardia due to pain, anxiety, hypovolemia, anemia, drug effect, or stimulation of the sympathetic nervous system. In patients undergoing non-cardiac surgeries tachycardia is rarely caused by a primary etiology. Atrial fibrillation can be seen in up to $60 \%$ of adult patients undergoing myocardial revascularization, and it usually peaks on the third postoperative day when inflammatory response 
is greater ${ }^{4}$. Development of supraventricular tachycardia (SVT) or non-sustained ventricular tachycardia (VT) can be secondary to hypoxemia, hypercarbia, acidosis, hypotension, electrolyte imbalance, mechanical irritation, pulmonary artery catheter, thoracic tube, hypothermia, micro- or macroshocks, adrenergic stimulation (e.g., superficial anesthesia), use of dysrhythmogenic drugs, and myocardial ischemia. Perioperative dysrhythmias are usually reversible and before being treated the most common causes should be excluded.

\section{Mechanisms of dysrhythmias}

Dysrhythmias are secondary to changes in cardiac ion channels (sodium, calcium, and potassium channels) and adrenergic receptors are the targets. To better understand the mechanism of dysrhythmias and antidysrhythmic agents, one should remember that the action potential is divided in five phases $(0 \text { to } 4)^{7}$. The initial period of the action potential corresponds to phase 0 and it initiates the conduction in the cardiac tissue. In atria and ventricles the impulse originates in the sodium current. In the sinoatrial (SA) and atrioventricular (AV) nodes phase 0 is produced by calcium current. Phases 1, 2, and 3 represent repolarization; the plateau (maintained by calcium current) is phase 2, and its end (phase 3 ) is maintained by potassium current. During phase 4 , nodal cells undergo spontaneous depolarization while atrial and ventricular tissues are hyperpolarized.

Dysrhythmias may be due to changes in the formation of the electric impulse (automaticity) or in conduction. Abnormal impulse generation can occur in the sinus node or in ectopic foci. Automaticity refers to abnormal atrial or ventricular depolarization during the repolarization (phases 2 or 3 ) or resting (phase 4) period of the action potential 6 . Some molecular substrates, such as prolongation of the QT interval and low potassium $\left(\mathrm{K}^{+}\right)$concentrations can trigger automaticity. Mutations in ion channels responsible for repolarization, and that can prolong it, make cardiac cells more sensitive to dysrhythmias ${ }^{8}$. Factors that increase automaticity include increased activity of the sympathetic nervous system, hypokalemia, hypomagnesemia, catecholamines, digoxin, hypoxemia, and atrial and ventricular dilation ${ }^{9}$. Besides the abnormal generation of impulses, abnormal conduction (reentry) is also responsible for dysrhythmias.

Three factors must be present for reentry to occur: 1) presence of two conduction pathways; 2) unidirectional blockade of one of the pathways prevents progression of the impulse, but is allows retrograde conduction; and 3) reduced impulse velocity in one of the pathways giving time for the other pathway to depolarize. Reentry is the mechanism of several supraventricular and ventricular dysrhythmias implying the presence of a pathologic circuit of electrical impulse around a functional or anatomic loop, which is seen in Wolff-Parkinson-White syndrome (WPW). Ischemia also predisposes the development of reentry tachycardia. Drugs that terminate reentry do this by two mechanisms: suppression of the current responsible for phase 0 of the action potential that prolongs or blocks conduction in the reentry pathway, interrupting the dysrhythmia. Drugs that prolong the action potential (with $\mathrm{K}^{+}$-channel blocking properties) prolong cellular and reentry circuit refractory period, blocking propagation of impulses through the circuit. Reentry is responsible for $90 \%$ of SVTs in children. The main mechanism of monomorphic VT is also reentry around the infracted myocardium.

\section{Risk factors for dysrhythmias}

Risk factors for the development of dysrhythmias can be classified as modifiable and non-modifiable. The non-modifiable risk factors include dilated cardiac diseases, ischemic cardiomyopathy, autonomic changes of the conduction system, polymorphism of ion channels, or congenital long-QT syndrome (LQTS). Among modifiable factors there are electrolyte changes: $\mathrm{K}^{+}$changes can generate increases in automaticity and abnormal formation of impulses. Changes in serum $\mathrm{K}^{+} \mathrm{le}-$ vels are closely related with the development of dysrhythmias, and abrupt changes are less tolerable than chronic changes. The relationship between preoperative $\mathrm{K}^{+}$and perioperative adverse events was demonstrated by Wahr et al. ${ }^{10}$, and serum $\mathrm{K}^{+}$levels below $3.5 \mathrm{mEq} . \mathrm{L}^{-1}$ can predict the development of perioperative dysrhythmias.

Magnesium is important for several physiologic functions activating ATPase and promoting transport of cations, such as calcium and potassium ${ }^{11}$. Severe hypomagnesemia increases automaticity and predisposes to the development of torsades de pointes (TdP). Although magnesium deficiency can contribute for several dysrhythmias, especially after cardiac surgeries 12,13 , and magnesium seems effective in decreasing catecholamine-induced dysrhythmias ${ }^{14,15}, \mathrm{TdP}$ is the only dysrhythmia in which magnesium has been proven to be effective ${ }^{16}$. Hypomagnesemia usually is concomitant with hypokalemia and hypocalcemia, making it difficult to adequately replace $\mathrm{K}^{+}$or calcium without replacing magnesium. Hypermagnesemia can cause bradycardia, first degree atrioventricular block, and increase the QT interval.

\section{Differential diagnosis of dysrhythmias}

Supraventricular tachycardia can be defined as sustained, non-sinus related, acceleration of the cardiac rhythm, originating above the $\mathrm{AV}$ node. On the other hand, autonomic tachycardia is rare and can be defined as a tachycardia initiated and sustained by an ectopic focus. Automatic atrial tachycardia is a type of automatic tachycardia that involves primarily the atrial tissue. The continuous type is usually symptomatic and often results from dilated cardiomyopathy. The repetitive type is frequently interrupted by periods of sinus rhythm, is less severe, and only becomes symptomatic during periods of very fast heart rate (HR).

There is a tendency to distinguish tachycardia as ventricular and supraventricular according to their origin. Patients with electrocardiographic changes compatible with wide-QRS re- 
gular tachycardia represent a diagnostic and treatment challenge. History, factors like history of AMI, physical exam, and electrocardiographic (ECG) findings, such as AV dissociation are useful to distinguish the origin of the tachycardia, although none of these factors is sensitive and specific. Adenosine is useful to classify SVTs because tachycardia caused by atrial reentry such as AF or flutter have transient response of decreased frequency after adenosine, but it does not terminate the dysrhythmia; on the other hand, SVTs due to reentry in the $\mathrm{AV}$ node ceases after adenosine. Ventricular dysrhythmias do not respond to adenosine because these dysrhythmias originate in distal tissues of the conduction system. This also allows the use of adenosine to distinguish between wide-complex SVT and VT, since adenosine causes rapid AV block, with a half-life of 9 seconds, and terminates most SVTs due to reentry; on the other hand, it causes transient AV block and is incapable of terminate most VT, but since adenosine has a brief effect, in case of VT it does not cause major problems.

However, the safety of adenosine has been questioned and, according to the 2005 guidelines of the American Heart Association ${ }^{17}$, it should not be used for differential diagnosis between wide-complex SVT and VT due to its vasodilator properties, bronchospasm, paradoxal increase in conduction in the accessory pathway, persistent bradycardia or asystole, and degeneration to ventricular fibrillation (VF). In contrast, in a study by Marril et al. ${ }^{18}$, in which consecutive patients were treated with adenosine between 1991 and 2006, the authors observed that adenosine is useful and safe to distinguish between VT and SVT in patients with stable and regular tachycardia. Besides, it has been postulated that in these cases adenosine would be useful to prevent treatment of VT with a long-acting $\mathrm{AV}$ node blocker, which would be risky since it causes vasodilation and longer-lasting preload reduction.

Non-sustained ventricular dysrhythmias can be divided according to their morphology (monomorphic or polymorphic) and duration (sustained or non-sustained). The main mechanism of monomorphic VT is the formation of a reentrant pathway around a tissue scar of a healed myocardial infarction. In monomorphic VT the amplitude of the QRS complex is constant, while in polymorphic VT the morphology of the QRS changes continuously. Non-sustained ventricular tachycardia (NSVT) is defined as three or more premature ventricular contractions with a frequency higher than 100 beats per minute (BPM) lasting 30 seconds or less without hemodynamic compromise. These dysrhythmias are routinely seen in the absence of cardiac disease and might not require drug therapy in the perioperative period. Non-sustained ventricular tachycardia is seen in approximately $50 \%$ of patients during or after large size cardiac or vascular surgeries and they do not affect late mortality in patients without ventricular dysfunction.

\section{Drugs that prolong the QT interval}

Normal QT interval measures up to 450 msecs. Approximately 60 medications increase the QT interval and can cause TdP. Type la (for example, quinidina) and type III antiarhyth- mics can cause TdP. Type Ib and Ic antiarhythmics rarely cause TdP ${ }^{19-25}$. Despite its ability to prolong the QT interval amiodarone only causes TdP in $1 \%$ of patients when compared to other type III drugs that can cause TdP in $2 \%$ to $4 \%$ of patients. Drugs that precipitate TdP delay repolarization, allowing formation of several reentry sites (Table I). Risk factors for induction of TdP include bradycardia, recent cardioversion of $A F$, heart failure, hypomagnesemia, hypokalemia, digoxin, rapid infusion of drugs that increase the QT interval, long-QT, female gender, LQTS, and ion channel polymorphism ${ }^{26,27}$. In patients receiving drugs that increase the QT interval, QT interval monitoring is indicated for prophylaxis of TdP 26,27. To correct the QT interval, which is influenced by $\mathrm{HR}$ and gender, and to find the corrected QT (QTC), Fridericia $(\mathrm{QTC}=\mathrm{QT} / 3 \mathrm{RR})$ or Bazett (QTC $=\mathrm{QT} / \mathrm{RR})$ formulas are used. Bazett correction is used more often, but it can generate hypercorrection in short RR intervals and hypercorrection in long RR intervals ${ }^{28}$.

\section{Prodysrhythmia}

A term used to describe antiarhythmic drug-induced dysrhythmia ${ }^{29}$. Vaughan Williams classification ${ }^{30}$ divides antiarhythmic drugs into four classes according to their effects on ion channels. Singh ${ }^{25}$ reviewed the drugs used to treat dysrhythmias and found that the use of class la drugs are declining; class Ib drugs such as lidocaine are still widely used in the perioperative period; while class Ic drugs should not be used in patients with structural cardiomyopathies due to the high risk

Table I - Drugs that Affect Repolarization, Prolong the QT Interval, with Documented Cases of Torsades de Pointes

\begin{tabular}{ll}
\hline Type of drug & Example \\
\hline Class la antiarhythmic & $\begin{array}{l}\text { Quinidine, disopyramide, } \\
\text { procainamide }\end{array}$ \\
Class Ic antiarhythmic & Flecainide \\
Class III antiarhythmic & Sotalol, amiodarone \\
Antipsychotics & $\begin{array}{l}\text { Droperidol, haloperidol, } \\
\text { phenothiazine }\end{array}$ \\
Antipsychotics & Thioridazine \\
"Atypical" antipsychotic & $\begin{array}{l}\text { Pimozide, quetiapine, } \\
\text { risperidone, zotepine }\end{array}$ \\
Serotonin reuptake inhibitors & Fluoxetine, paroxetine, sertraline \\
Macrolide antibiotics & Erythromicin, clarythromicin, \\
& azythromicin \\
5-HT1 agonists & Zolmitriptan, naratriptan \\
Antimalarial agents & Halofantrine \\
Antihistamines & Terfenadine \\
Prokinectic agents & Cisapride \\
\hline
\end{tabular}


of prodysrhythmia. In patients after myocardial infarction slow conduction due to class Ic antiarhythmics leads to an increase in reentry allowing the development of VT and, therefore, class Ic drugs should not be used in patients with ischemia. Class la drugs can also slow down conduction and prolong repolarization, and may cause TdP. Procainamide, sotalol, and bipyridyl can cause TdP. Some antiarhythmic drugs such as verapamil and amiodarone can occasionally lead to TdP, although amiodarone prolongs the QT interval by more than 500 msecs and, despite this, the development of TdP is rare.

Class Ib drugs (lidocaine and mexiletine) are more selective to abnormal or damaged myocytes and are not associated with prodysrhythmia. Class III agents (amiodarone, sotalol, and dofetilide) block potassium channels prolonging repolarization, and have the potential to induce TdP. Although this is observed with sotalol and dofetilide, it rarely occurs with amiodarone, which is considered safe and neutral in patients after myocardial infarction. Amiodarone also has class II (blocking adrenergic receptors) and class IV (calcium blockers) properties and it can cause bradycardia. Beta blockers (class II) may be used to treat SVTs, as well as calcium channel blockers (class IV), and they do not have a great risk of prodysrhythmic phenomena. Class III drugs such as amiodarone and sotalol prolong the action potential and, currently, are replacing class I drugs due to the inherent risk of these drugs for causing dysrhythmias. In reality, all antiarhythmic drugs have the potential to generate dysrhythmias and for this reason care should be taken when using them, especially in patients with cardiac abnormalities and electrolytic changes ${ }^{31,32}$.

\section{Dysrhythmia related to drugs used in the perioperative period}

Propofol, nitrous oxide $\left(\mathrm{N}_{2} \mathrm{O}\right)$, and sevoflurane have little arrhythmogenic potential and very few side effects ${ }^{31}$. Halothane is not a good choice in the presence of dysrhythmias. Isoflurane causes ventricular dysrhythmias in $2.5 \%$ of patients, while desflurane increases the HR. Sevoflurane, halothane, and isoflurane can delay ventricular repolarization and prolong the QT interval. Propofol does not change the HR, has little effect on cardiac conduction, and, whether it is associated with a negative inotropism, this is due to a reduction in sympathetic tonus and increase in parasympathetic sensitivity. Ketamine causes node dysrhythmia and decreases contractility, but the HR can increase.

Opioids except meperidine reduce the HR through a central mechanism that reduces sympathetic tonus and increases vagal tonus ${ }^{31}$. Fentanyl has a direct effect on SA node. Midazolam has a biphasic effect on HR, since it affects the sympathetic and parasympathetic nervous system and its effects on dysrhythmias are not clear. Bronchodilators stimulate adrenergic receptors, increasing the risk of cardiovascular events; in patients with baseline tachycardia these drugs have the potential to exacerbate dysrhythmias. Although levalbuterol has been developed to avoid the cardiovascular effects of albuterol, tachydysrhythmias still occur in $2.7 \%$ of patients. Drugs that induce bradydysrhythmias include betablockers, calcium channel blockers, amiodarone, clonidine, and dexmedetomidine. Bradycardia is seen in $5 \%$ of patients with dexmedetomidine and this agent should be avoided in patients with heart block.

Droperidol has been associated with ventricular dysrhythmias ${ }^{32}$ and in 2001 it was placed on the Food and Drug Administration's (FDA) black list, as it is associated with prolonged QT interval and malignant dysrhythmias such as TdP. The relative risk of dysrhythmias with droperidol compared to other antiemetic drugs or placebo is not clear; therefore, recommendation for droperidol requires 12-lead ECG before administration and continuous ECG monitoring for 2 to 3 hours after administration. If the QTc is prolonged in baseline ECG, droperidol administration is not recommended. Extreme caution is recommended when using droperidol in patients with risk factors for prolonged QT interval, such as congestive heart failure, bradycardia, use of diuretics, ventricular hypertrophy, hypokalemia, hypomagnesemia, or use of drugs that prolog the QT interval. Droperidol should be initiated in low doses and adjusted until the desired results are obtained.

Inotropics, such as dobutamine and milrinone, may precipitate arrhythmias and ventricular fibrillation. Dobutamine is directly dysrhythmogenic, causing dose-dependent tachycardia; doses higher than $5 \mu \mathrm{g} \cdot \mathrm{kg}^{-1} \cdot \mathrm{min}^{-1}$ are prone to cause dysrhythmias and generate little benefit in oxygen transportation. Milrinone increases the inotropism without activating adrenergic receptors, but it also has a dysrhythmic potential. Digoxin increases intracellular calcium in cardiac myocytes, slows AV node conduction, and can increase automaticity, triggering any type of dysrhythmia, the most common being premature ventricular contraction, heart blocks of any degree (although rarely Mobitz type II), paroxysmal atrial tachycardia with heart block, accelerated junctional rhythm, and bidirectional VT. Risk factors for digoxin intoxication include renal dysfunction and electrolyte imbalance, such as hypokalemia, hypomagnesemia, and hypercalcemia. Drugs that cause electrolyte imbalances have the potential to cause dysrhythmias and are on Table II.

\section{Treatment of dysrhythmias}

Although severe bradyarhythmias require treatment they have been reported in only $0.4 \%$ of 17,021 patients undergoing general anesthesia. These patients respond well to pharmacological measures or transesophageal atrial pacemaker and rarely required permanent transvenous pacemaker even in the presence of asymptomatic bifascicular block or preoperative left branch block ${ }^{33}$. On the other hand, it has been estimated that perioperative tachyarhythmias affect almost one million elderly Americans, being associated with significant morbidity. A large number of patients undergoing cardiac or non-cardiac surgeries have these dysrhythmias. Because all antiarhythmics have the potential to cause dysrhythmias they 
Table II - Drugs that Cause Electrolyte Abnormalities

\begin{tabular}{llll}
\hline Hypokalemia & Hyperkalemia & Hypomagnesemia & Hypermagnesemia \\
\hline Beta-adrenergic agonists & Non-steroidal anti-inflammatories & Thiazide diuretics & Magnesium-containing laxatives \\
Catecholamines & $\mathrm{K}^{+}$-sparing diuretics & Loop diuretics & Parenteral nutrition \\
Insulin & Succinylcholine & Aminoglycosides & Lithium \\
Loop diuretics & Digoxin & Amphotericin B & \\
Theophylline & Angiotensin-converting enzyme & Cisplatin \\
inhibitors & \\
Amiazide diuretics & Angiotensin receptor blockers & Cyclosporine \\
Amphotericin B & Beta blockers & Digoxin \\
Mineralocorticoid & & Mannitol & Methotrexate \\
\hline
\end{tabular}

Table III - Electrophysiological Changes of Anesthetics

\begin{tabular}{lll}
\hline Drug & Action & Effects \\
\hline Inhalational & $\begin{array}{l}\text { Antagonize calcium and increase depolarization in } \\
\text { Purkinje fibers }\end{array}$ & $\begin{array}{l}\text { Junctional rhythm, atrioventricular } \\
\text { asynchrony }\end{array}$ \\
Propofol & Stimulate muscarinic receptors & Bradycardia \\
Succynilcholine & Activate muscarinic or nicotinic receptors & $\begin{array}{l}\text { Tachycardia or bradycardia, it can lead to } \\
\text { asystole }\end{array}$ \\
Pancuronim & Increase catecholamines and automatism & Tachycardia \\
Vecuronium & Decrease automatism by sympathetic blockade & Bradycardia and junctional rhythm \\
Local Anesthetics & Block calcium channels & Widening of the QRS, Tachycardia, and VF \\
Opioids & Decrease frequency of the SA node & Bradycardia \\
& Prolong AV conduction & \\
Ketamine & Increase SA node frequency by sympathetic & Tachycardia \\
Clonidine and Dexmedetomidine & activation & Bradycardia \\
\hline
\end{tabular}

Table IV - Interaction between Anesthetics and Antiarhythmics

\begin{tabular}{ll}
\hline Adenosine & $\begin{array}{l}\text { Vasodilation with isoflurane and neuraxial block, } \\
\text { Bronchoconstriction with neostigmine, } \\
\text { Asystole with neostigmine, dexmedetomidine and opioids, } \\
\text { Antagonism with aminophylline }\end{array}$ \\
Amiodarone & Myocardial depression and vasodilation with inhalational agents \\
Digoxin & Bradycardia is potentiated by halothane and succinylcholine, \\
& Care should be taken when administering calcium and using diuretics (hypokalemia) \\
Beta blocker & Myocardial depression is potentiated by halothane, \\
& Bronchoconstriction with neostigmine and atracurium \\
Quinidine & Prolongs the effects of neuromuscular blockers (NMB) \\
Procainamide & Antagonizes neostigmine, \\
Calcium channel blocker & Ventricular dysrhythmias when combined with phenotiazides \\
Magnesium & Bradycardia and myocardial depression with halogenated agents and dantrolene, \\
Lidocaine & Potentiates NMB \\
\hline
\end{tabular}


should be used with caution, and correction of electrolyte imbalances and prevention of bradycardia are required in the management of dysrhythmias ${ }^{33}$.

\section{Sinus tachycardia}

Tachycardia increases myocardial oxygen consumption generating ischemic episodes and increasing mortality, therefore, it should be treated. Beta blockers can be used for this purpose and during anesthesia drugs with short half-life and continuous infusion such as esmolol should be used.

Recently, Landiolol has been introduced into clinical practice in some countries. It is a beta-blocker whose cardioselective properties are greater than the esmolol and has a shorter half-life (2 to 4 minutes), as it is rapidly hydrolyzed by plasma esterases. Harasawa et al. ${ }^{34}$ administered Landiolol to treat tachycardia during anesthesia and to obtain protection against myocardial ischemia to evaluate its dose-dependent bolus effects in doses of $0.1,0.2$, or $0.3 \mathrm{mg} \cdot \mathrm{kg}^{-1}$. They did not observe hypotension, bradycardia, or ischemic changes on ECG with $0.1 \mathrm{mg} \cdot \mathrm{kg}^{-1}$, and for this reason the authors suggested that this would be the optimal dose to reduce the effects of tachycardia. On the other hand, higher doses such as $0.3 \mathrm{mg} \cdot \mathrm{kg}^{-1}$ caused a reduction in $\mathrm{HR}$ and blood pressure and they can be used in patients with tachycardia and hypertension in response to surgical stress ${ }^{34}$. In a case report by Chrysostomou et al. ${ }^{35}$ of dexmedetomidine $0.3 \mathrm{mg} \cdot \mathrm{kg}^{-1}$ used to treat persistent sinus tachycardia resistant to treatment with esmolol the authors found no side effects, therefore, they suggested that this drug could be useful in patients with bronchospasm and tachycardia.

\section{Supraventricular tachycardia (SVT)}

Perioperative SVTs should be initially considered as a sign of potentially life-threatening underlying clinical condition. Therefore, the initial conduct is to look for an underlying cause, usually procedure-related, and possible repercussions. These conditions are often reversible and antiarhythmics drugs should be considered only after these etiologies have been ruled out. Another fundamental step in the management of dysrhythmias in general is recognizing symptoms of dysrhythmia-related hemodynamic instability such as hypotension, changes in the level of consciousness, thoracic pain, or any other sign of poor tissue perfusion. In such cases, electrical cardioversion is the initial conduct.

Remember that perioperative cardioversion may not have maximum effectiveness or even may not be able to maintain an organized rhythm for adequate time, taking into consideration that cardioversion itself does not reverse the baseline cause of dysrhythmias. But cardioversion is indicated in any case of tachydysrhythmia with hemodynamic repercussions and in the perioperative period it can be useful in that, during a small period of sinus rhythm, there might be time to initiate measures to reverse the baseline cause 7 . A study of patients with SVTs (especially AF) undergoing cardiac surgery with extracorporeal circulation demonstrated that electrical lowenergy cardioversion was effective in $80 \%$ of cases, but the recurrence rate after 1 minute was greater than $50 \%{ }^{36}$. It has been recommended, when deciding for perioperative elective electrical cardioversion that initially an attack dose of an antiarhythmic agent should be administered to minimize the recurrence of SVT after the shock. Adenosine is the drug of choice to treat SVTs involving the SA or AV node $(6 \mathrm{mg}$ IV rapid flush, which can be repeated as $12 \mathrm{mg}$ bolus), important especially in cases of node reentry, which is not the main mechanisms of perioperative SVTs.

Most patients with perioperative SVTs maintain hemodynamic stability and, therefore, they do not need immediate electrical cardioversion. For this reason, heart rate control is the most important conduct in the treatment of this disorder; for this end, AV node blockers, such as beta blockers and calcium channel blockers (class II or IV), are used. Among IV beta-blockers, esmolol, due to its pharmacologic characteristics (short half-life and easy titration) is the drug of choice in the perioperative period. Verapamil and diltiazem also promote fast ventricular rate control in SVTs. Intravenous diltiazem has less negative inotropic effects when compared with verapamil, being preferable in patients with heart failure. In these patients, diltiazem, digitalis, and amiodarone are the recommended drugs to control heart rate.

A prospective randomized study compared the efficacy of diltiazem versus amiodarone to control HR in patients with atrial tachycardia and $\mathrm{HR}>120 \mathrm{bpm}$. Diltiazem showed better HR control than amiodarone; however, it was associated with a higher rate of hypotension ${ }^{37}$. In patients with history of SVT due to the presence of an anomalous pathway (WPW) the use of $\mathrm{AV}$ node blockers is contraindicated due to the increased risk of developing malignant ventricular dysrhythmias. These rugs accentuate the refractory period of the anomalous pathway. In this case, it is possible to use procainamide and amiodarone ${ }^{7}$. Chemical cardioversion of these perioperative dysrhythmias has little significance, being reserved for the cases of failure to control heart rate or absence of reversion and hemodynamic instability with electrical cardioversion. The efficacy of antiarhythmics in cardioversion is questioned in several studies, as several patients showed reversion of the rhythm with the use of placebo in randomized studies. A randomized clinical assay demonstrated $60 \%$ conversion in 24 hours in the placebo group compared with $68 \%$ in the amiodarone group ${ }^{38}$.

Even knowing that with high doses of amiodarone the rate of chemical conversion is considerable, the potential for postoperative side effects should be considered to establish the risk-benefit ratio. Drugs like procainamide and amiodarone may be useful to treat any of those dysrhythmias, but they may be ineffective in some cases, in addition to having adverse effects, especially procainamide currently used in exceptional cases.

Atrial fibrillation is well tolerated in young patients, but it can be harmful in elderly patients, and it should be aborted whenever possible. Statins have been tested in the preoperative period to reduce the incidence of peri- and postoperative 
AF and they can represent important adjuvant therapy in the prevention of this dysrhythmia ${ }^{39}$. Amiodarone can also be effective after cardiac surgeries, although a study questioned its prophylactic efficacy post-thoracotomy. In case of AF, strategies to reduce $\mathrm{HR}$ should be adopted in the first 24 hours as more than $85 \%$ of the episodes will resolve within this period. Class Ic or III antiarhythmics can be used.

Regarding prophylaxis of $\mathrm{AF}$, a perioperative cardiopathy very common in cardiac surgeries, Beaulieu et al. ${ }^{40}$ in a randomized prospective study of 120 patients who received amiodarone or placebo for prevention of AF in this type of surgery concluded that amiodarone was not effective to prevent $A F$.

\section{VENTRICULAR TACHYCARDIA (VT)}

\section{Non-sustained ventricular tachycardia (NSVT)}

It is a common perioperative occurrence and in the absence of cardiac disease it does not require treatment. In patients with cardiopathies this non-sustained rhythm can predict malignant ventricular dysrhythmias. The main strategy in NSVT would be prevention, instituting immediate treatment when possible risk factors arise ${ }^{41}$. A study of patients undergoing cardiac surgery demonstrated that correction of magnesium after ECC reduced the incidence of non-sustained VT ${ }^{12}$.

\section{Sustained ventricular tachycardia}

It is divided into two categories: monomorphic or polymorphic. Lidocaine, procainamide, or amiodarone are used in monomorphic VT ${ }^{42}$. The management in polymorphic ventricular tachycardia is based on the prior presence of a prolonged QT interval and consequent development of TdP. In such cases, the treatment of dysrhythmia consists of reversing the prolonged QT interval and especially discontinuation of drugs that can cause increased QT.

The approach of TdP may include cardioversion, although magnesium is the treatment of choice $(2 \mathrm{~g}$ administered IV slowly). Because TdP is usually recurrent, efforts should be made to increase HR between 150 and 120 bpm through pacemaker or inotropic drugs. Drugs that prolong repolarization time such as procainamide are contraindicated during TdP. Discontinuation of drugs that prolong the QT interval and correction of electrolyte is usually necessary. In refractory high risk patients, one should consider placing a pacemaker and defibrillator. In cases in which there is doubt if polymorphic VT is the result of prolonged QT interval, it is recommended using a channel blocking drug and replace magnesium empirically ${ }^{7}$. Among the drugs that prolong the QT interval, the incidence of
TdP with amiodarone is smaller; therefore, amiodarone IV is a good choice as an alternative therapy for refractory polymorphic VT of unknown etiology.

\section{Unstable VT, pulseless VT, VF}

The main maneuvers for patients with perioperative unstable VT, pulseless VT, or VF are not pharmacological. They are immediate defibrillation, cardiopulmonary resuscitation (CPR) in case of cardiorespiratory arrest, and correction of reversible causes.

\section{CONCLUSION}

After the diagnosis of dysrhythmia the distinction between benign dysrhythmia and those that carry the risk of sudden death is fundamental. The choice of anesthetic agents is important to minimize episodes of tachydysrhythmias. Prevention is as important as treating dysrhythmias. Recognition of risk factors, adequate selection of drugs for each patient, and monitoring represent the most important steps in prevention. In patients at risk for prolonged QT, recent baseline ECG is important. Electrolytes should be monitored and corrected whenever necessary. Verification of renal and liver function is important in patients using medications that can cause dysrhythmias. Moreover, doses should be adjusted whenever necessary. Prolonged QT interval should be closely monitored by the anesthesiologist, as several drugs can potentially prolong it, and the interaction among them can be catastrophic. Supraventricular tachycardia responds to treatment with adenosine, while AF does not, although in both cases the frequency is controlled by beta-blockers or calcium channel blockers. The use of class la drugs is gradually declining due to its unfavorable risk/benefit ratio. Class lb drugs such as lidocaine are still widely used in the operating room to treat ventricular tachydysrhythmias. Class lc drugs should not be used in patients with structural cardiac disease due to the high risk of proarhythmia. Beta-blockers or calcium channel blockers may be used in SVTs when the aim is reducing HR. Amiodarone and sotalol are class III drugs and they prolong the action potential and repolarization, and are currently among the most used antiarhythmics drugs. Digoxin, magnesium, and adenosine have unique antidysrhythmic properties - and digoxin is rarely indicated in the perioperative period. Magnesium and adenosine, on the contrary, have been increasingly used in the preoperative period, having specific indications that should be followed. The management in perioperative dysrhythmias does not always include the use of antiarhythmics agents, although the use of antiarhythmic drugs should not be delayed when indicated. 